\title{
Family Medicine and Community Health \\ Factors for self-assessment score of interprofessional team collaboration in community hospitals in Japan
}

\author{
Junji Haruta, ${ }^{1}$ Sachiko Ozone, ${ }^{2}$ Ryohei Goto ${ }^{2}$
}

To cite: Haruta J, Ozone S, Goto R. Factors for selfassessment score of interprofessional team collaboration in community hospitals in Japan. Fam Med Com Health 2019;7:e000202. doi:10.1136/fmch-2019-000202

Check for updates

(C) Author(s) (or their employer(s)) 2019. Re-use permitted under CC BY-NC. No commercial re-use. See rights and permissions. Published by BMJ.

${ }^{1}$ Department of Primary Care and Medical Education, Faculty of Medicine, University of Tsukuba, Tsukuba, Japan ${ }^{2}$ Department of General Medicine and Primary Care, Faculty of Medicine, University of Tsukuba, Tsukuba, Japan

Correspondence to

Dr Junji Haruta;

junharujp@md.tsukuba.ac.jp

\section{ABSTRACT}

Objective Interprofessional collaboration is considered an important strategy in overcoming the complex issues associated with healthcare outcomes. A nationwide, community-based integrated care system developed for the care of older people in individual communities in Japan requires community hospitals to deliver integrated care to coordinate efforts for creating effective environments for health. This study aimed to explore the factors associated with the self-assessment score of interprofessional collaboration in community hospitals.

Design Cross-sectional study using the Assessment of Interprofessional Team Collaboration Scale (AITCS).

Setting This study was conducted in three small community hospitals in Japan.

Participants All healthcare staff in the hospitals via research collaborators were asked to complete the anonymous self-administered questionnaire of the AITCS comprising questions related to individual factors (age, gender, profession), hospital to which they belonged, relationships with neighbouring facilities, job burden and job satisfaction from July to October 2018. The association between the questionnaire items as explanatory variables and AITCS score as an objective variable was determined using univariate followed by multiple regression analyses.

Results The data from 325 of 630 participants were analysed, of whom 252 were female $(77.5 \%)$ and 240 were nurses $(73.8 \%)$. The mean of the total AITCS score was 117.6 (range 37-185), and the Visual Analogue Scale for relationships with neighbouring facilities, job satisfaction and job burden was $53.0 \mathrm{~mm}(0-100)$, $46.1 \mathrm{~mm}(0-100)$ and $64.3 \mathrm{~mm}(0-100)$, respectively. In univariate analyses of the association with AITCS score, explanatory variables with a significance level of $<0.05$ were relationships with neighbouring facilities, job satisfaction and job burden. On multiple regression analysis, the total AITCS score was independently associated with age, profession (nurse/non-nurse), relationships with neighbouring facilities and job satisfaction.

\section{Conclusions Better self-assessment score of} interprofessional collaboration is more strongly associated with younger age, a nursing profession, better relationships with neighbouring facilities and greater job satisfaction than with the hospital to which the participant belonged. These findings may help community hospitals enhance the integration of service delivery and benefit to the community through interprofessional collaboration.

\section{INTRODUCTION}

Interprofessional collaboration (IPC) is considered an essential strategy for improving the efficiency of healthcare systems and health outcomes. Policy makers, implementers and educators are aware of the need for cooperation among medical professionals to improve health outcomes, healthcare quality and medical safety. ${ }^{1}$ In particular, given the increase in complex healthcare needs of individuals and populations in our rapidly changing society, policy makers in many countries have focused on advancing care delivery and enhancing collaboration in primary care. ${ }^{2-4}$ Canada has implemented diverse policies, including primary care payment blended models that are mostly group-oriented. To restructure individual practices around interprofessional care, the integrated and interprofessional team model can provide resources in a blended capitationbased or salary-based model. ${ }^{5}$ In the UK, the 'Integrated Care Pioneer' programme has provided multidisciplinary approaches to meeting the needs of the ageing population, delivering person-centred, coordinated and long-term support. The multidisciplinary care teams have provided better healthcare, and have in turn reduced the cost of local healthcare provision. ${ }^{6}$ Similarly, the USA formulated the Patient-Centered Medical Home model to transform the organisation and delivery of advanced primary care services. ${ }^{7}$ The model coordinates care across the elements of the broader healthcare system and is expected to improve quality and decrease the cost of care. ${ }^{8}$

In Japan, the government has introduced a comprehensive and integrated nationwide, community-based integrated care system to care for older people with chronic diseases or disabilities. ${ }^{9}$ In particular, in cross-sectional/organisational collaboration, healthcare professionals in community hospitals should collaborate with specialists 
and staff in advanced treatment hospitals, and with staff in clinics and welfare facilities, to facilitate smooth hospital discharge by sharing patients' and families' background information. ${ }^{10}$ As a community hub for health and social services, community hospitals should commit to both the process of IPC and patients' flow and outcome in delivering integrated care at the community level. ${ }^{11}$ However, a number of challenges are associated with developing and maintaining interprofessional networks within and across organisations. ${ }^{12}{ }^{13}$ Meanwhile, few studies have examined the factors related to IPC in community hospitals.

This study aimed to explore the factors associated with the self-assessment score of IPC in community hospitals with consideration for the effect of confounding factors.

\section{METHODS \\ Design and setting}

The study was conducted under a cross-sectional design using an anonymous self-administered questionnaire.

Surveys were conducted at three primary-level hospitals in Ibaraki Prefecture in Japan. Two hospitals (hospitals A and $\mathrm{B}$ ) are private, while the third (hospital C) is a public healthcare institution, and all are located 1-2 hours by car from the city centre. The hospitals have 199, 179 and 183 beds, respectively. ${ }^{14}$ The cities where the three hospitals are located have a population of 30000,90000 and 40 000 , respectively. The three hospitals provide communitybased and primary care-led service-level care, ${ }^{11}$ including a few specialties (mainly internal medicine, paediatrics and general surgery) and general practice, with limited laboratory services, available for general but not specialised pathological analysis. All three hospitals have played a key role in a community-based integrated care system for communication among clinics, welfare facilities, and secondary or tertiary hospitals.

\section{Participants}

We distributed the anonymous self-administered questionnaire to all healthcare staff via research collaborators in the three community hospitals. Study participants included all staff in the three hospitals, excluding those who did not participate in providing healthcare services to patients, such as clerks, engineers and cleaners, and those who did not consent to participate in the study, because we aimed to focus on collaboration within interprofessional team practices with patient involvement. The questionnaires were collected from July to October 2018.

\section{Variables}

The objective variable was the Assessment of Interprofessional Team Collaboration Scale (AITCS) score. AITCS is a validated measurement tool developed in Canada for evaluating collaboration within teams across various practice settings and the integration of patient involvement as part of team practice. ${ }^{15}$ In its original form, it consists of
37 items and 3 subscales representing discrete elements of interprofessional care, including (1) partnership/ shared decision making, (2) cooperation and (3) coordination. The Japanese version of the AITCS was verified for reliability and cultural adaptability in $2018 .{ }^{16}$ This verification identified three corresponding subscales based on factor analysis: 'Patient-centered collaborative care', 'Teamwork among healthcare professionals' and 'Patient participation'.

The explanatory variables were selected by reviewing the literature and considering the effect of IPC in community hospitals. First, we defined age, gender and healthcare profession as individual factors. These factors are associated with implicit bias in healthcare settings. ${ }^{17}$ Typically, since nurses tend to adopt a more collaborative culture than other professionals, ${ }^{18} 19$ we analysed healthcare profession data by dividing subjects into nurse and non-nurses (other) professions. Additionally, hospital culture can have a marked effect on collaboration. ${ }^{20}$ To meet the community's needs, there is also a growing requirement for IPC both within and across organisations. ${ }^{21}$ Such interorganisational/ intraorganisational collaboration can be organised according to individual multidisciplinary teams across the boundaries of different organisations and sectors. ${ }^{22}$ Based on this concept, we selected the hospital to which staff belonged and relationships with neighbouring facilities as other variables. We estimated the neighbouring facilities included hospitals or welfare facilities which are located in the same medical administration area for participants' hospital. Moreover, the merits associated with IPC include job satisfaction, ${ }^{23} 24$ while disadvantages include emotional burden or collaboration burden regarding other professional relationships. ${ }^{25} 26$ Therefore, participants' characteristics (age, gender, healthcare profession and hospital to which they belonged), relationships with neighbouring facilities (determined using the Visual Analogue Scale (VAS)), job burden (VAS) and job satisfaction (VAS) were selected as explanatory variables. This explains why VAS has better responsiveness than the Likert scale and appears to be more closely associated with what participants experience. ${ }^{27}$ For the VAS, participants were asked to mark the point on a $100 \mathrm{~mm}$ horizontal line, with the end points clearly marked, that was representative of their feeling towards the variable in question. The distance from one end of the line to the participant's mark was measured and used as a quantitative variable for statistical analysis.

\section{Statistical analyses}

We examined the distribution of each explanatory variable. After determining the descriptive statistics, we investigated the association between the explanatory variables and the objective variable, namely the total AITCS score. First, univariate analyses were performed using Pearson's correlation coefficient. With consideration for age, gender, profession and hospital as potential confounders, 


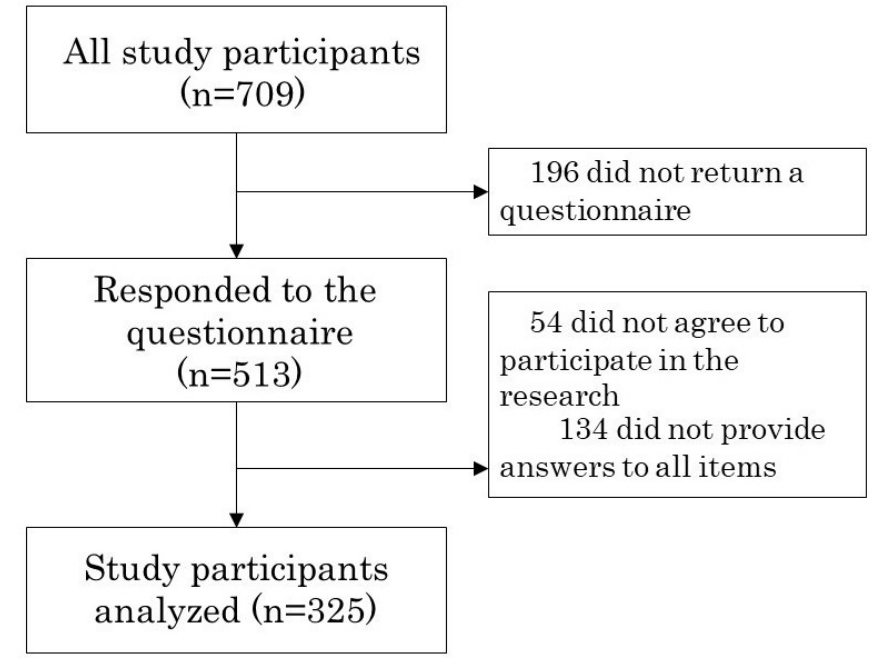

Figure 1 Study participant flow.

variables found to be significant by univariate analysis were considered for multiple regression analysis. To eliminate potential multicollinearity, significant explanatory variables were reviewed based on the correlation coefficients of similar variables to determine which to include in the multiple regression analysis. All statistical analyses were performed using SPSS V.24 statistical software. All $\mathrm{p}$ values were two-sided and considered significant at $\mathrm{p}<0.05$.

\section{Sample size}

For multiple regression analyses, the desired level is between 15 and 20 observations for each predictor variable. ${ }^{28}$ Accordingly, more than 240 samples were targeted per hospital in anticipation of a $50 \%$ response rate.

\section{RESULTS}

A total of 826 questionnaires were distributed, equivalent to the total number of staff in the three participating hospitals. Of the 709 participants surveyed, 196 did not return the questionnaire, while the remaining 513 responded. Of these, 54 did not agree to participate in the research and 134 did not provide answers to all items, leaving 325 participants for analysis (figure 1). Among these, $77.5 \%$ were female and the average age was 39.6 years. A total of 240 were nurses $(73.8 \%), 24$ were doctors $(7.4 \%), 16$ were rehabilitation therapists $(4.9 \%)$ and 12 were pharmacists $(3.7 \%)$ (table 1$)$. There were $148(45.5 \%)$ participants from hospital A, $111(34.1 \%)$ from hospital B and $66(20.3 \%)$ from hospital C. Among the 325 participants, the mean \pm SD of the total AITCS score was 117.6 \pm 25.3 (range 37-185), and the VAS for relationships with

Table 1 Participant characteristics $(\mathrm{N}=325)$

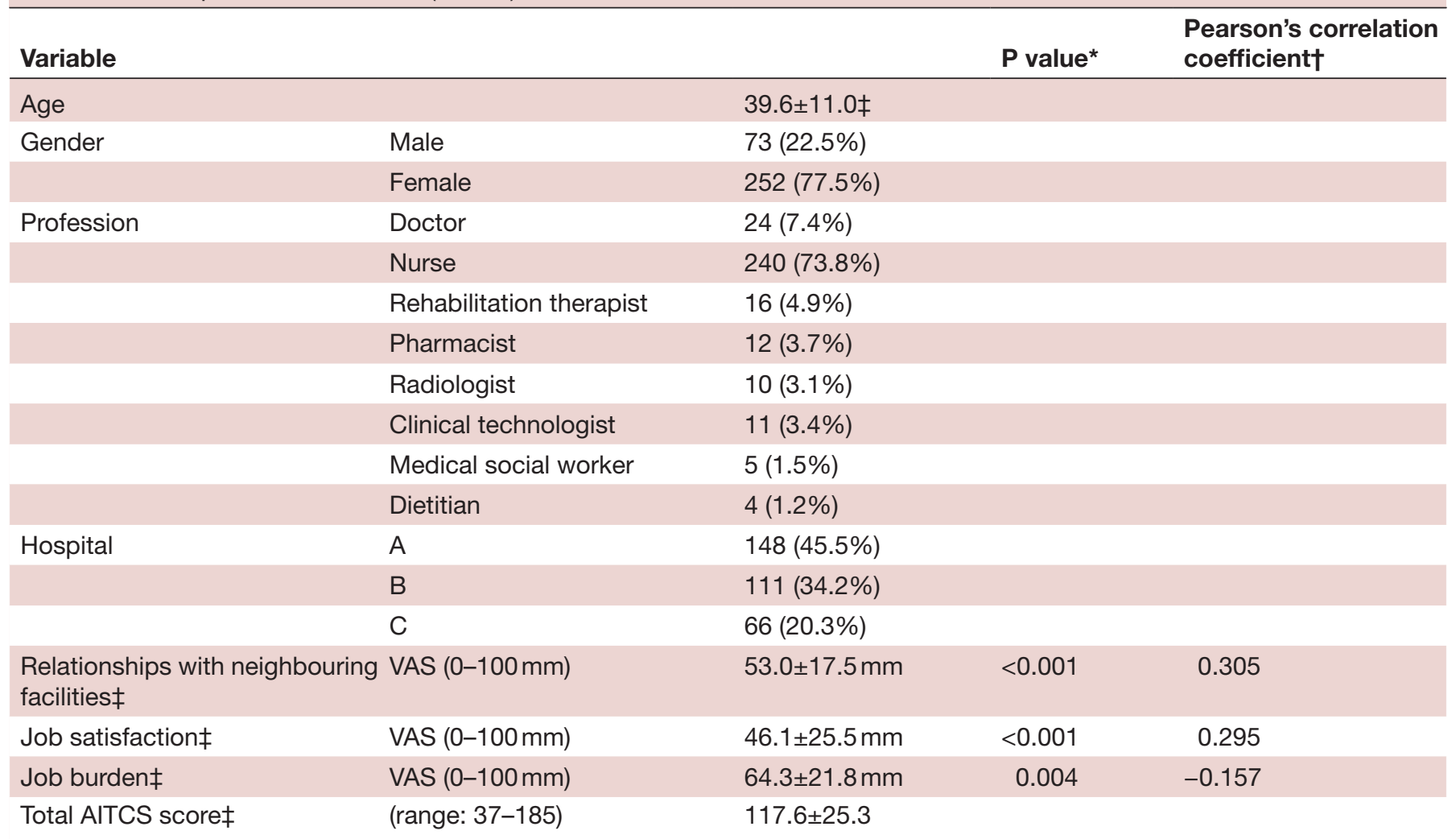

*Univariate analysis of the association with AITCS score (Pearson's correlation coefficient). †Pearson's correlation coefficient between age or VAS and individual AITCS scores. $\ddagger$ Mean \pm SD.

AITCS, Assessment of Interprofessional Team Collaboration Scale; VAS, Visual Analogue Scale. 
Table 2 Comparison of variables among hospitals A, B and C ( $N=325)$

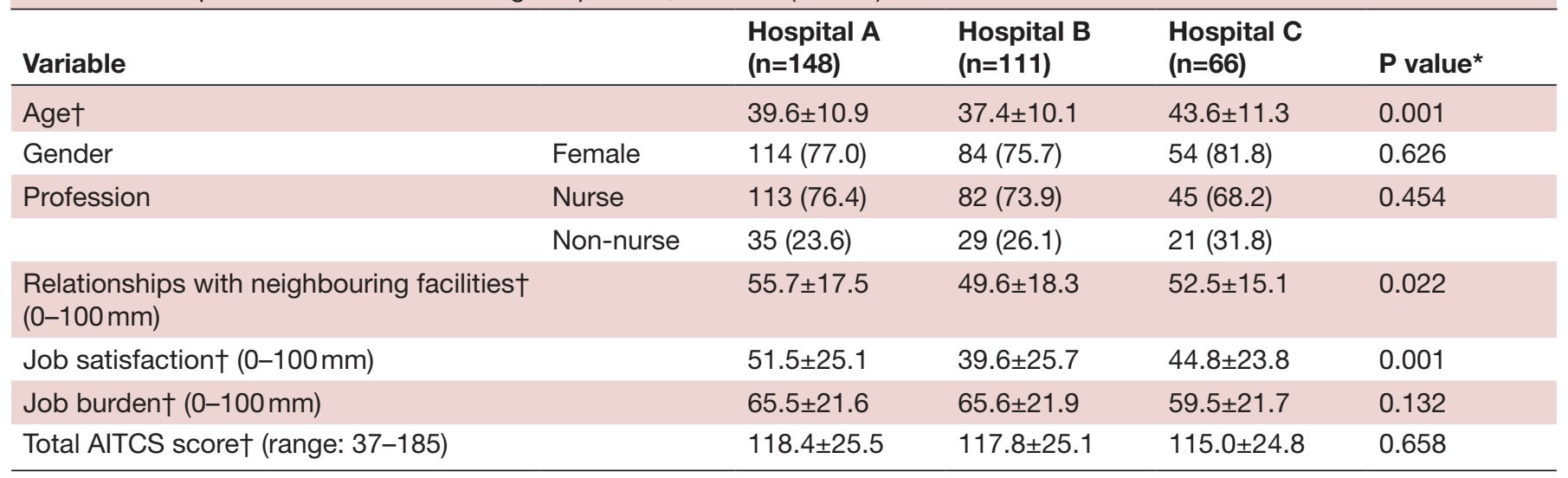

${ }^{*} \chi^{2}$ test or analysis of variance.

†Mean \pm SD.

AITCS, Assessment of Interprofessional Team Collaboration Scale.

neighbouring facilities, job satisfaction and job burden was $53.0 \pm 17.5 \mathrm{~mm}(0-100), 46.1 \pm 25.5 \mathrm{~mm}(0-100)$ and $64.3 \pm 21.8 \mathrm{~mm}(0-100)$, respectively (table 1$)$. Age, relationships with neighbouring facilities and job satisfaction were statistically significantly different among hospitals A, $\mathrm{B}$ and $\mathrm{C}$ (table 2).

\section{Univariate analyses}

The results of univariate analyses of the association between AITCS score and explanatory variables are presented in table 1. Explanatory variables with a significance level of $<0.05$ were relationships with neighbouring facilities $(\mathrm{r}=0.305, \mathrm{p}<0.001)$, job satisfaction $(\mathrm{r}=0.295, \mathrm{p}<0.001)$ and job burden $(\mathrm{r}=-0.157, \mathrm{p}=0.004)$. No significant correlation was observed between any two of these variables. After univariate analyses, we checked the frequency distribution of each variable and confirmed that there was no ceiling or floor effect. All three variables were therefore further examined using multiple regression analysis.

\section{Multiple regression analysis}

Multiple regression analysis was performed using an analytical model that included the following explanatory variables: age, gender, profession (nurse or non-nurse), hospital to which the staff belonged (A, B and $\mathrm{C}$ ), relationships with neighbouring facilities, job satisfaction and job burden. The results are presented in table 3 . The beta coefficients $(\beta)$ of age, profession (reference, nurse), relationships with neighbouring facilities and job satisfaction in the AITCS total score were $-0.196(95 \%$ CI -0.68 to -0.22 , $\mathrm{p}<0.001), 0.156$ (95\% CI 1.96 to $16.0, \mathrm{p}=0.012), 0.263(95 \%$ CI 0.230 to $0.530, \mathrm{p}<0.001)$ and $0.224(95 \%$ CI 0.11 to 0.33 , $\mathrm{p}<0.001)$, respectively.

\section{DISCUSSION}

The AITCS score, a self-assessment score of IPC, was associated with age, profession (nurse/non-nurse), relationships with neighbouring facilities and job satisfaction. Our findings suggest that younger professionals or nurses may be ideal members to lead community hospitals in increasing opportunities for IPC among clinics, welfare facilities, and secondary or tertiary hospitals, and to enhance self-efficacy-associated job satisfaction. Given that evidence on the effectiveness of community

\begin{tabular}{|c|c|c|}
\hline $\boldsymbol{\beta}$ & P value* & $95 \% \mathrm{Cl}$ \\
\hline-0.196 & $<0.001$ & -0.680 to -0.223 \\
\hline 0.156 & 0.012 & 1.961 to 15.976 \\
\hline 0.039 & 0.440 & -2.002 to 4.596 \\
\hline 0.224 & $<0.001$ & 0.113 to 0.330 \\
\hline-0.015 & 0.783 & -0.143 to 0.108 \\
\hline & $<0.001$ & 80.142 to 114.957 \\
\hline
\end{tabular}

$r=0.474, r^{2}=0.225$, adjusted $r^{2}=0.208$.

${ }^{*}$ Multiple regression analysis of the association with AITCS score. Bold text indicates a statistically significant correlation with a $p$-value less than 0.05 .

AITCS, Assessment of Interprofessional Team Collaboration Scale. 
hospitals is relatively scarce, these findings may be useful for systematic changes in community hospitals, which can play the role of community hub to provide health and social services based on a more locally integrated health and care system.

Younger age was associated with higher total AITCS score. This is inconsistent with previous reports which suggest that it is typically difficult for novice professionals to play the role that other professionals envision of them within their own profession and to understand the contextual roles of other professions. ${ }^{29}{ }^{30}$ IPC requires that mutual professionals learn expected roles with, from and about each other through prolonged interprofessional communication across many years. Based on their prerequisite education, it is likely that young healthcare professionals may regard superficial sharing of information as IPC, ${ }^{31}{ }^{32}$ suggesting that younger healthcare professionals may tend to think that they conduct better IPC than older healthcare professionals. Younger professionals who are motivated to collaborate more within and across organisations may play a critical role in IPC.

Nurses also play a critical role in IPC ${ }^{33}$ and should therefore be more autonomous in IPC. ${ }^{34}$ Additionally, nurses can lead other professionals in effective IPC and create a culture that encourages the values and role models of collaborative practice within a team context. ${ }^{35}$ These characteristics of nurses may affect the self-assessment score of IPC.

Relationships with neighbouring facilities reflect interorganisational relationships. To enhance the effectiveness of interorganisational relationships, communication should be formal and informal within and across organisations. ${ }^{34}$ Additionally, because the degree of organisational relationships is linked to levels of collaboration, effective relationships across organisations should be active, regular, reciprocal, open and comfortable for communication among members. ${ }^{36}$ Such open and flexible communication styles within or across organisations may promote effective horizontal and vertical IPC. Horizontal integration refers to peer-based and cross-sectoral collaboration to improve overall health, while vertical integration refers to that which transcends organisational boundaries and connects community-based generalists with largely hospital-site specialists. ${ }^{10}$ Both types of collaboration can interact with each other and break down the walls of multiprofessionals and multiorganisations.

Other studies have shown that job satisfaction is associated with interprofessional perspective,${ }^{37}$ team climate, ${ }^{38}$ and organisational culture and teamwork. ${ }^{39}{ }^{40}$ While a number of previous studies have demonstrated that organisational culture is important for IPC,${ }^{41}{ }^{42}$ we found that relationships with neighbouring facilities were more strongly associated with IPC than the hospital to which participants belonged in multiple regression analysis. Moreover, job satisfaction was independently associated with IPC even after accounting for confounding factors. That healthcare professionals in community hospitals may regard IPC as their role may explain the association of the self-assessment score of IPC with job satisfaction. ${ }^{41}$

This study has several limitations. First, confounding factors of AITCS were defined as participants' characteristics, namely age, gender, healthcare profession and hospital to which they belonged, and potential associated factors were relationships with neighbouring facilities, job burden and job satisfaction. It is likely that there were other confounding factors given that IPC is used as a dimension of organisational culture, ${ }^{43}$ leadership is a dimension of teamwork, and climate is highly associated with culture. ${ }^{39}$ However, this study showed that factors associated with the organisational culture of different hospitals were not independently associated with IPC in multiple regression analysis. Second, this study focused on only separating nurse and non-nurses (other) as profession categories. A larger sample is more representative of the population and needed to analyse detail factors (more professional categories, and the size of clinics and hospitals, and so on). Third, the findings may not be generalisable to all community-based hospitals. However, comparison of the low response rate with that in other studies in healthcare ${ }^{27}$ suggests that these findings may be useful for developing an interprofessional education system in community hospitals. Given that evidence on the effectiveness of community hospitals is relatively scarce, a strength of our study is that our findings may be useful for systematic changes in community hospitals to enhance the integration of service delivery and benefit to the community. In particular, Japan has a unique work culture comprising a hierarchical and relationshipdependent climate within organisations, and workers are expected to be hard working. Given that this cultural background may differ among countries, it is important to compare factors associated with IPC in other cultures.

\section{CONCLUSION}

Better self-assessment score of IPC was associated with younger age, a nursing profession, better relationships with neighbouring facilities and greater job satisfaction

\section{Key points}

- To create a community-integrated care system through interprofessional collaboration (IPC), the present study aimed to explore the factors associated with IPC in community hospitals in Japan.

- We concluded that IPC may be associated with younger age, a nursing profession, better relationships with neighbouring facilities and greater job satisfaction, and these factors were more strongly associated with IPC than the organisation to which participants belonged in multiple regression analysis.

- Given the lack of evidence on the effectiveness of community hospitals, the value of this study lies in its identification of factors associated with IPC in community hospitals.

- These findings are useful for systematic changes in community hospitals, which can play the role of community hub to provide health and social services based on a more locally integrated health and care system. 
in community hospitals in Japan. These findings may be useful for helping community hospitals to play the role of community hub to provide health and social services based on a more locally integrated health and care system.

Contributors $\mathrm{JH}, \mathrm{SO}$ and GR were involved in the conception and design of this study, carried out all qualitative enquiries, analysed the data and wrote the paper.

Funding The authors have not declared a specific grant for this research from any funding agency in the public, commercial or not-for-profit sectors.

Competing interests None declared.

Patient consent for publication Not required.

Ethics approval This study was approved by the Ethics Committee of the University of Tsukuba (approval number: 1202-3).

Provenance and peer review Not commissioned; externally peer reviewed.

Data availability statement The datasets during and/or analysed during the current study available from the corresponding author on reasonable request.

Open access This is an open access article distributed in accordance with the Creative Commons Attribution Non Commercial (CC BY-NC 4.0) license, which permits others to distribute, remix, adapt, build upon this work non-commercially, and license their derivative works on different terms, provided the original work is properly cited, appropriate credit is given, any changes made indicated, and the use is non-commercial. See: http://creativecommons.org/licenses/by-nc/4.0/.

\section{REFERENCES}

1 Jackson GL, Powers BJ, Chatterjee R, et al. The patient-centered medical home: a systematic review. Ann Intern Med 2013;5:169-78.

2 Stange KC, Nutting PA, Miller WL, et al. Defining and measuring the patient-centered medical home. J Gen Intern Med 2010;25:601-12.

3 The Conference Board of Canada. Improving Primary Health Care Through Collaboration: Briefing 1 - Current Knowledge About Interprofessional Teams In Canada [Internet]. Briefing October HealtH HealtH Care and Wellness 2012 http://www.wrha.mb.ca/ professionals/collaborativecare/files/CBCBriefing22012.pdf

4 Elston S, Holloway I. The impact of recent primary care reforms in the UK on interprofessional working in primary care centres. $J$ Interprof Care 2001;15:19-27.

5 Sweetman A, Buckley G. Ontario's Experiment with Primary Care Reform. SPP Reserach Pap 2014;7:1-38.

6 Department of Health. Transforming primary care: safe, proactive, personalised care for those who need it most. NHS England and health education England 2014.

7 Quality A for HR and Agency for Healthcare Research and Quality. Patient Centred Medical Home Resource Centre [Internet]. Available: https://pcmh.ahrq.gov/ [Accessed 14 Apr 2019].

8 Assurance TNC for Q. The National Committee for Quality Assurance: Delivery system reform. Available: https://www.ncqa.org/ public-policy/ [Accessed 14 Apr 2019].

9 Hatano $\mathrm{Y}$, Matsumoto M, Okita M, et al. The vanguard of communitybased integrated care in Japan: the effect of a rural town on national policy. Int J Integr Care 2017;17:2.

10 Thomas P, Meads G, Moustafa A, et al. Combined horizontal and vertical integration of care: a goal of practice-based commissioning. Qual Prim Care 2008;16:425-32.

11 Pitchforth E, Nolte E, Corbett J, et al. Community hospitals and their services in the NHS: identifying transferable learning from international developments - scoping review, systematic review, country reports and case studies. Heal Serv Deliv Res 2017.

12 Zaheer A, McEvily B, Perrone V. Does trust matter? exploring the effects of Interorganizational and interpersonal trust on performance. Organization Science 1998;9:141-59.

13 Oliveria SA, Lapuerta P, McCarthy BD, et al. Physician-related barriers to the effective management of uncontrolled hypertension. Arch Intern Med 2002;162:413-20.

14 Foreign Language. / ibaraki-prefecture [Internet]. Available: http:// www.pref.ibaraki.jp/site/foreign-language/index.html [Accessed cited 2019 Jun 14]

15 Orchard CA, King GA, Khalili H, et al. Assessment of interprofessional team collaboration scale (AITCS): development and testing of the instrument. J Contin Educ Health Prof 2012;32:58-67.
$16 \mathrm{Yu}$ Y, Junji H. Reliability and cultural adaptation of Assessment of Interprofessional Team Collaboration Scale (AITCS) in Japanese. In: All together better health IX, 2018.

17 FitzGerald C. A neglected aspect of conscience: awareness of implicit attitudes. Bioethics 2014;28:24-32.

18 Karam M, Brault I, Van Durme T, et al. Comparing interprofessional and interorganizational collaboration in healthcare: a systematic review of the qualitative research. Int J Nurs Stud 2018;79:70-83.

19 Finn R, Learmonth M, Reedy P. Some unintended effects of teamwork in healthcare. Soc Sci Med 2010;70:1148-54.

20 Mulvale G, Embrett M, Razavi SD. 'Gearing Up' to improve interprofessional collaboration in primary care: a systematic review and conceptual framework. BMC Fam Pract 2016;17.

21 Axelsson R, Axelsson SB. Integration and collaboration in public health - a conceptual framework. Int J Health Plann Manage 2006;21:75-88.

22 Willumsen E, Ahgren B, Ødegård A. A conceptual framework for assessing interorganizational integration and interprofessional collaboration. J Interprof Care 2012;26:198-204.

23 Chang W-Y, Ma J-C, Chiu H-T, et al. Job satisfaction and perceptions of quality of patient care, collaboration and teamwork in acute care hospitals. J Adv Nurs 2009;65:1946-55.

24 K.J.O'Leary SNL, Terrell G, Williams MV. Interdisciplinary teamwork in hospitals: a review and practical recommendations for improvement. J Hosp Med 2012;7:48-54.

25 Skei K. Collaboration at risk: registered nurses' experiences on orthopaedic wards. J Clin Nurs 2008;17:1907-14.

26 Cross R, Gray P. Where has the time gone? addressing collaboration overload in a Networked economy. Calif Manage Rev 2013;56:50-66.

27 Hasson D, Arnetz BB. Validation and findings comparing vas vs. Likert scales for psychosocial measurements. Int Electron J Health Educ 2005;8:178-92.

28 Siddiqui K. Heuristics for sample size determination in multivariate statistical techniques. World App/ Sci J 2013;27:285-7.

29 Witt Sherman D, Maitra K, Gordon Y, et al. Illustrating and analyzing the processes of interprofessional collaboration: a lesson learned from palliative care in Deconstructing the concept. J Palliat Med 2017;20:227-34

30 Moss E, Seifert PC, O'Sullivan A. Registered nurses as interprofessional collaborative partners: creating value-based outcomes. J Issues Nursing 2016;21.

31 Goto M, Haruta J, Oishi A, et al. A cross-sectional survey of interprofessional education across 13 healthcare professions in Japan. TAPS 2018;3:38-46.

32 Maeno T, Haruta J, Takayashiki A, et al. Interprofessional education in medical schools in Japan. DeBaets am, editor. PLoS One 2019;14:e0210912

33 Timmins F. Nursing Research Generating and Assessing Evidence for Nursing Practice. In: Nurse education in practice. , 2013: 13, e29.

34 Matziou V, Vlahioti E, Perdikaris $\mathrm{P}$, et al. Physician and nursing perceptions concerning interprofessional communication and collaboration. J Interprof Care 2014;28:526-33.

35 Wong CA, Cummings GG, Ducharme L. The relationship between nursing leadership and patient outcomes: a systematic review update. J Nurs Manag 2013;21:709-24.

36 Bradley F, Ashcroft DM, Noyce PR. Integration and differentiation: a conceptual model of general practitioner and community pharmacist collaboration. Res Social Adm Pharm 2012;8:36-46.

37 Konrad TR, Fletcher GS, Carey TS. Interprofessional collaboration and job satisfaction of chiropractic physicians. J Manipulative Physiol Ther 2004:27:245-52.

38 Espinoza P, Peduzzi M, Agreli HF, et al. Interprofessional team member's satisfaction: a mixed methods study of a Chilean hospital. Hum Resour Health 2018;16.

39 Körner M, Wirtz MA, Bengel J, et al. Relationship of organizational culture, teamwork and job satisfaction in interprofessional teams. BMC Health Serv Res 2015;15.

40 Hartnell CA, Ou AY, Kinicki A. Organizational culture and organizational effectiveness: a meta-analytic investigation of the competing values framework's theoretical suppositions. J Appl Psychol 2011;96:677-94.

41 Aiken LH, Sloane DM, Bruyneel L, et al. Nurses' reports of working conditions and hospital quality of care in 12 countries in Europe. Int $J$ Nurs Stud 2013:50:143-53.

42 Cameron A. Impermeable boundaries? developments in professional and inter-professional practice. J Interprof Care 2011;25:53-8.

43 Scott T, Mannion R, Davies $\mathrm{H}$, et al. The quantitative measurement of organizational culture in health care: a review of the available instruments. Health Serv Res 2003;38:923-45. 